\title{
Non-cooperative RSU Deployment in Vehicular Networks
}

\author{
Ilario Filippini*, Francesco Malandrino ${ }^{\dagger}$, György Dán ${ }^{\ddagger}$, Matteo Cesana*, Claudio Casetti ${ }^{\dagger}$, Ian Marsh ${ }^{\S}$ \\ *Politecnico di Milano, Dipartimento di Elettronica ed Informazione, Email: \{ilario.filippini|cesana\}@elet.polimi.it \\ ${ }^{\dagger}$ Politecnico di Torino, Dipartimento di Elettronica, Email: \{malandrino|casetti $\}$ tlc.polito.it \\ ${ }^{\ddagger}$ KTH Royal Institute of Technology, School of Electrical Engineering, Email: gyuri@ee.kth.se \\ $\S$ Swedish Institute of Computer Science, Email: ianm@sics.se
}

\begin{abstract}
This work considers the issue of distributing contents to vehicles through roadside communication infrastructure. Within this scenario, this work studies the dynamics of infrastructure deployment by using game theoretic tools. A strategic game is used to model the case in which the operators perform their deployment decisions concurrently, whereas an extensive game is used to study the dynamics in case one operator is the deployment leader and moves first. The equilibria of the aforementioned games are then assessed as a function of several parameters (nominal infrastructure capacity, interference, vehicle flows). Simulations are used to validate the analytical findings.
\end{abstract}

\section{INTRODUCTION}

In this paper, we focus on the vehicle-to-infrastructure (V2I) communication paradigm for VANETs and try to provide insights into one of the recurring problems therein, i.e., that of information exchange with passing vehicles. Specifically, we assume that a road segment must be equipped for information dissemination through the deployment of infrastructure nodes, called Road Side Units (RSUs). We also assume that vehicles are equipped with embedded communication devices, hereinafter named On-Board Units (OBU), capable of exchanging information content with RSUs. In such a scenario, we ask ourselves the following question: if competing providers wish to select locations where to deploy their RSUs in order to provide or collect data to/from passing vehicles, what kind of strategies should they follow? The answer, predictably, depends on several factors: vehicle density, data traffic patterns and sizes, presence of incumbent operators, to name a few.

Several works in the literature have addressed the problem of the deployment of RSUs for vehicular access. Basic RSU deployments have been proposed with the aim of enhancing connectivity in urban VANETs [1], and for delay-tolerant routing among vehicles [2], or targeting timely content dissemination [3], [4]. Alternatively, the formulation in [5] targets a minimum coverage guarantee, while the one in [6] maximizes the minimum contact opportunity between vehicles and RSUs. Finally, the work in [7] aims at finding the RSU deployment that maximizes the throughput while accounting for airtime conflicts due to the presence of an arbitrary number of vehicles. Unlike the works above, where the RSU infrastructure is owned by a single operator, we aim at studying the dynamics of scenarios where different operators may competitively deploy their RSUs to attract the largest number of customers.
In the present work, without purporting to provide a comprehensive solution, (i) we set the problem of RSU deployment that maximizes the revenue for a content provider within the framework of game theory; (ii) we derive preliminary results, that can be extended for a more general approach to the problem and (iii) we verify the validity of our approach through simulation.

\section{REFERENCE SCENARIO DESCRIPTION}

We consider a scenario with two operators $\mathrm{O}_{1}$ and $\mathrm{O}_{2}$, which would like to deploy Road Side Units (RSUs) for distributing content along a stretch of road of length $D$. Each operator can deploy its RSUs at a subset of a set of candidate sites $J$. Each RSU is characterized by a coverage range $R$, which defines its service area, and by an applicationlevel goodput $c$ for content delivery. The goodput depends on the wireless technology the RSU is equipped with and on the communication protocols used for content delivery. Furthermore, in practice the goodput might be affected by physical layer impairments, interference, and collision with other transmissions to/from the same or different RSUs.

We account for such impairments in the form of inter-RSU interference, which is a function of the distance $d$ between the interfering RSUs, and determines the goodput that the individual RSUs can achieve. We express the goodput of each RSU at distance $d$ as

$$
c(d)=\frac{c}{1+\omega(d)},
$$

where the interference function $\omega(d)$ is a monotone nonincreasing function of $d$. The interference function is bounded, $\Omega=\omega(0) \geq \omega(d) \geq \omega(D) \geq 0$ for $0 \leq d \leq D$. The assumption of monotone non-increasingness is rather reasonable, since the interference level usually does not increase when interferers move away. Furthermore, we consider the relevant case when $\Omega>1$. As an example, if two RSUs are deployed at the same candidate location then they could use a MAC protocol to share the physical medium, and their total capacity would be $\frac{2 c}{1+\Omega}<c$.

There is a bidirectional flow of vehicles on the considered stretch of road; $\lambda_{A}$ is the intensity of the flow of vehicles from left to right, and $\lambda_{B}$ is the intensity from right to left. The vehicles move at some constant speed $v[\mathrm{~m} / \mathrm{s}]$. Each vehicle aims to retrieve some content with an average size 


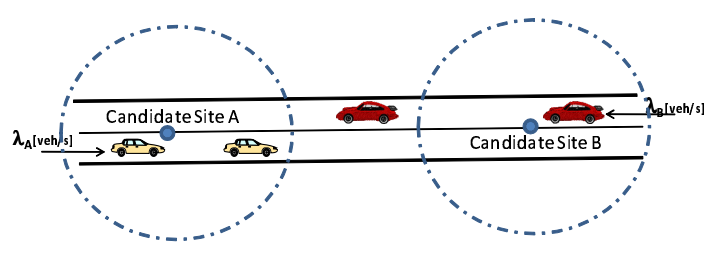

Fig. 1. Reference scenario with two candidate sites at the two extremes of the stretch of road.

of $S$ [bits], Depending on the content size, multiple MAC layer frames may be required to accomplish the content download. Content retrieval is attempted from the first met RSU along the road upon completion of a standard association procedure. In case multiple RSUs (from different operators) are available simultaneously, the RSU association is done at random. A content download is successful if the vehicle manages to retrieve the content before leaving the coverage area of the RSU. If the content retrieval is unsuccesful, the vehicle attempts to download the content via the next RSU encountered along the road. We define the offered load as $\rho_{A}=\lambda_{A} S$ and $\rho_{B}=\lambda_{B} S$ in the two directions, respectively. This definition of load does not consider factors such as the number of vehicles in the coverage area, the content size, or the ratio of successful content retrievals, but it is appropriate for our purposes. We consider that the revenue of an operator in a deployment is proportional to the traffic load it serves, that is, to the number of vehicles that successfully get service through the operator's RSU. Figure 1 shows a scenario with two candidate sites for RSU deployment $(J=\{A, B\})$ at the two extremes of a stretch of road.

\section{RSU DEPLOYMENT GAMES}

We consider a scenario where operators $i(i \in\{1,2\})$ can deploy a single RSU each at one of two candidate locations $A$ and $B$ at distance $D$, as shown in Fig. 1. The goal of each operator is to maximize its own revenue. We consider that $D$ is large enough so that the interference between RSUs deployed at different candidate sites is negligible, i.e., $\omega(D)=0$. Our results can be easily generalized to non-zero interference, and as we will see, even the simple case of two locations gives rise to a rich set of solutions.

Clearly, the deployment choice of operator $O_{1}$ influences the revenue of operator $\mathrm{O}_{2}$, and vice versa, and the operators' choices influence what portion of the offered traffic will be served. Let us denote by $\rho_{A}^{u}\left(\rho_{B}^{u}\right)$ the unserved traffic offered by vehicles traveling from left to right (right to left). If the two RSUs are colocated then the operators share a total revenue of $\max \left[\rho_{A}+\rho_{B}, \frac{2 c}{1+\Omega}\right]$, and the unserved traffic is $\rho_{A}^{u}=\rho_{B}^{u}=$ $\max \left[0,\left(\rho_{A}+\rho_{B}-\frac{2 c}{1+\Omega}\right) / 2\right]$. Consider now that the RSUs are not colocated, and denote by $\rho_{A}^{s}\left(\rho_{B}^{s}\right)$ the spill-over offered traffic after passing the first RSU location.

We can consequently define the socially optimal RSU deployment as the deployment that minimizes the sum of the unserved traffic, i.e., $\rho_{A}^{u}+\rho_{B}^{u}$.

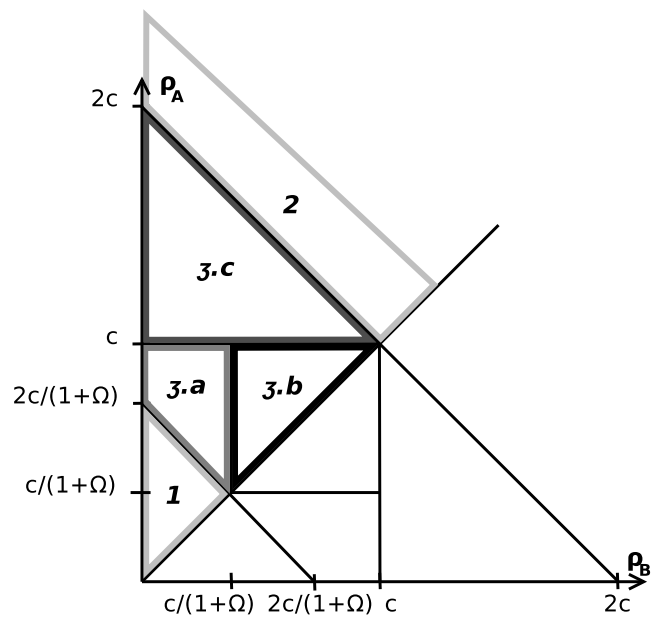

Fig. 2. $\left(\rho_{A}, \rho_{B}\right)$-space partition.

\section{A. Simultaneous Deployment}

Let us consider first that the two operators make their deployment choices simultaneously, based on the traffic loads $\rho_{A}$ and $\rho_{B}$. We can model the problem as a strategic game and we are interested in the efficiency of the Nash Equilibria (NE) of the game, which is quantified by the Price of Anarchy (PoA), i.e., the ratio of the total revenue in social optimum and the smallest total revenue in any NE. For the case of simultaneous deployment we can state the following.

Proposition 3.1: For the RSU deployment game the price of anarchy is: $P o A \leq 1+\Omega$.

Proof: In the case when the traffic intensity is symmetric, $\rho_{A}=\rho_{B}$, the equilibria are easy to obtain. If $\frac{2 c}{1+\Omega} \geq \rho_{A}+$ $\rho_{B}$ then any deployment is a NE, while colocation is not an equilibrium otherwise. Furthermore, all equilibria are socially optimal, hence $P o A=1$.

In the case when the traffic intensity is asymmetric the number and efficiency of the equilibria depend on the relationship between the offered traffic $\rho_{A}, \rho_{B}$, and the RSU capacity $c$. Without loss of generality we can assume that $\rho_{A}>\rho_{B}$. For convenience, let us divide the $\left(\rho_{A}, \rho_{B}\right)$-space in three partitions, as shown in Fig. 2: (1) $\rho_{A}+\rho_{B}<\frac{2 c}{1+\Omega}$, (2) $\rho_{A}+\rho_{B}>2 c$, and (3) $\frac{2 c}{1+\Omega}<\rho_{A}+\rho_{B}<2 c$. Partition (3) can further be divided into three partitions. In the following, we analyze the equilibria for the resulting five partitions shown in Fig. 2.

1) Colocation Underload (Partition 1): The total capacity of the RSUs is higher than the total offered traffic even under colocated deployment, i.e., $\frac{2 c}{1+\Omega}>\rho_{A}+\rho_{B}$. The NE is $(A, A)$, and the operators have equal revenues. Note that in the equilibrium there is no unserved traffic $\left(\rho_{B}^{u}=0, \rho_{A}^{u}=0\right)$, hence the NE is socially optimal.

2) Overload (Partition 2): The offered traffic is higher than the combined RSU capacity, i.e., $2 c<\rho_{A}+\rho_{B}$. In this case for any $\Omega>0$ there are two $\mathrm{NE},(A, B)$ and $(B, A)$. To see why, note that in both NE $\rho_{B}^{u}>0$ and $\rho_{A}^{u}>0$. Since both RSUs are fully utilized no player could benefit from colocation. Furthermore, both NE are socially optimal because 
each operator obtains a revenue $c$, which is greater than $\frac{c}{1+\Omega}$ with colocation.

3) Colocation Overload (Partition 3.a): The total offered traffic exceeds the capacity of colocated RSUs, a colocated RSU can serve one flow entirely, whereas the other flow requires the capacity of a non-colocated RSU, i.e., $\rho_{A}<c$, $\rho_{B}<\frac{c}{1+\Omega}, \rho_{A}+\rho_{B}>\frac{2 c}{1+\Omega}$, In this case $(A, A)$ is the unique NE. Observe that under colocation both operators would obtain $\frac{c}{1+\Omega}$ revenue, while under non-colocation one operator would obtain $\rho_{B}<\frac{c}{1+\Omega}$ (because $\rho_{A}^{s}=0$ ). In this case the NE is not socially optimal, as non-colocation, which is not a NE, gives a higher revenue, $\rho_{A}+\rho_{B}$. The price of anarchy is

$$
P_{o} A=\frac{\left(\rho_{A}+\rho_{B}\right)(1+\Omega)}{2 c}<1+\Omega,
$$

the inequality holds as $\rho_{A}+\rho_{B}<c+\frac{c}{1+\Omega}<2 c$.

4) Colocation Overload (Partition 3.b): The offered traffic from both directions exceeds the capacity of colocated RSUs but both flows can be served by non-colocated RSUs, that is, $\frac{c}{1+\Omega}<\rho_{B}, \rho_{A}<c$. In this case there are two NE, $(A, B)$ and $(B, A)$. In both NE all traffic is served, hence the NE are socially optimal and $\operatorname{PoA}=1$.

5) Asymmetric Overload (Partition 3.c): The traffic from left to right exceeds the RSU capacity but the total offered traffic is less than the total RSU capacity, i.e., $\rho_{A}>c$ and $\rho_{A}+\rho_{B}<2 c$.

The game admits different equilibria depending on the values of $\rho_{A}, \rho_{B}$, and $\Omega$. We can identify two regions in partition (3.c). Region I where $\rho_{A}>c$ and $\rho_{A}+\rho_{B}<c+\frac{c}{1+\Omega}$ and Region II where $c+\frac{c}{1+\Omega}<\rho_{A}+\rho_{B}<2 c$. Following similar arguments as for the previous cases we can state the following.

Lemma 3.2: In the case of asymmetric overload the NE are

$$
\mathrm{NE}= \begin{cases}\{(A, A)\} & \text { if }\left(\rho_{A}, \rho_{B}\right) \in \text { Region I } \\ \{(A, B),(B, A)\} & \text { if }\left(\rho_{A}, \rho_{B}\right) \in \text { Region II }\end{cases}
$$

The NE $(A, B)$ and $(B, A)$ are socially optimal, so for the price of anarchy we can state the following.

Lemma 3.3: In the case of asymmetric overload the price of anarchy is

$$
\mathrm{PoA}= \begin{cases}\frac{\left(\rho_{A}+\rho_{B}\right)(1+\Omega)}{2 c} & \text { if }\left(\rho_{A}, \rho_{B}\right) \in \text { Region I } \\ 1 & \text { if }\left(\rho_{A}, \rho_{B}\right) \in \text { Region II }\end{cases}
$$

Since $\rho_{A}+\rho_{B}<2 c$ we have $\mathrm{PoA} \leq 1+\Omega$, which concludes the proof.

\section{B. Leader/Follower Deployment}

Let us now consider the scenario where one of the two operators is the market leader and has the first-move advantage. We can model the problem as an extensive-form game and we are interested in its sub-game perfect NE. The NE derived in Section III-A for partitions 1, 2, and 3.a in Fig. 2 can be easily shown to be sub-game perfect. Nevertheless, not all NE in partitions 3.b and 3.c are sub-game perfect.

1) Colocation Overload (Partition 3.b): From the two NE $(A, B)$ and $(B, A)$ only $(A, B)$ is sub-game perfect. Indeed, the two NE have revenues $\left(\rho_{A}, \rho_{B}\right)$ and $\left(\rho_{B}, \rho_{A}\right)$, respectively. Since, $\rho_{A}>\rho_{B}$, operator $O_{1}$ will deploy its RSU in $A$, thus, the best choice for operator $O_{2}$ will be to choose $B$, and hence the revenue for the first-mover $O_{1}$ is greater than in $(B, A)$.
2) Asymmetric Overload (Partition 3.c): Given our definition of the revenue, the following can be verified.

Lemma 3.4: In the case of asymmetric overload the subgame perfect NE are

$$
\mathrm{NE}= \begin{cases}\{(A, A)\} & \text { if }\left(\rho_{A}, \rho_{B}\right) \in \text { Region I } \\ \{(A, B)\} & \text { if }\left(\rho_{A}, \rho_{B}\right) \in \text { Region II }\end{cases}
$$

Finally, we note that the price of anarchy in the case of leader/follower deployment equals that of the simultaneous deployment. This can be easily seen by comparing the set of NE to the set of sub-game perfect NE.

\section{Performance Evaluation}

In this section, we validate the analytical model via simulations in the ns-3 simulator. All vehicles travel at the constant speed of $20 \mathrm{~m} / \mathrm{s}$. After reaching the opposite end of the road, each vehicle is removed from the simulation. We make the conservative assumption that vehicles' OBUs communicate with RSUs using IEEE 802.11a at the basic rate of $6 \mathrm{Mb} / \mathrm{s}$, regardless of the distance from the RSU, and that the coverage area of an RSU is $200 \mathrm{~m}$. The mobility model is chosen to be the ns- 3 constant velocity model.

Upon transiting under the coverage of RSUs, an OBU first listens for their beacon (transmitted every second), then tries to associate with one of them (picked randomly if more than one beacon is received). Finally, if successful, it starts uploading its content to the selected RSU by using MAC frames that can carry $1 \mathrm{kB}$ of application data. If the transfer completes before the vehicles leaves the RSU coverage, the transfer is marked as successful. Otherwise, it counts as a failure, and the OBU will try to repeat the procedure upon coming under the coverage of another RSU (if any).

We consider two scenarios. The colocated scenario refers to both RSUs occupying the same candidate site (either A or B) and transmitting on the same channel. The disjoint (noncolocated) scenario is the one shown in Fig. 1, where the candidate sites are $600 \mathrm{~m}$ apart. In each plot we denote by "loc. A, alone" (resp. "loc. B, alone") the performance of the RSU in location A (resp. B) in the disjoint scenario; by "colocated" the performance of one RSU in the colocated scenario; and by "tot. disjoint" (resp. "tot. colocated")the performance of the two RSUs in the disjoint (resp. colocated) scenario.

We first study the case where the ratio of the left-to-right and right-to-left vehicle arrival rates (i.e., $\lambda_{A} / \lambda_{B}=10$ ) is fixed. In Fig. 3 we plot the number of successfully transmitted contents (whose size is fixed at $500 \mathrm{kB}$ for each vehicle). It is interesting to observe that, with these settings, whoever occupies location A first has the upper hand at low to medium traffic intensities. These results match the model predictions for the Colocation Underload case.

If the vehicle arrival rate increases, colocation is not a good choice for the newcomer, who fares better on its own, i.e., noncolocation becomes the NE as predicted in the Overload case. Interestingly, the disjoint solution provides an advantage for the incumbent who selected location A only up to $\left(\lambda_{A}<1.5\right.$ veh/s): at higher rates, its RSU cannot serve all vehicles and 


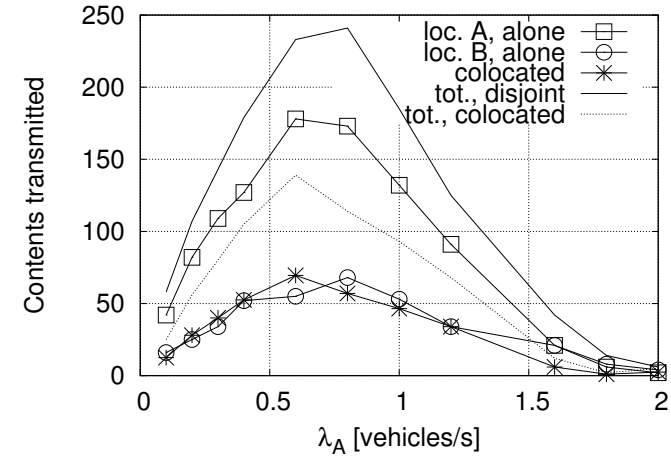

Fig. 3. Constant arrival imbalance: successful transmissions as a function of left-to-right vehicle flow intensity

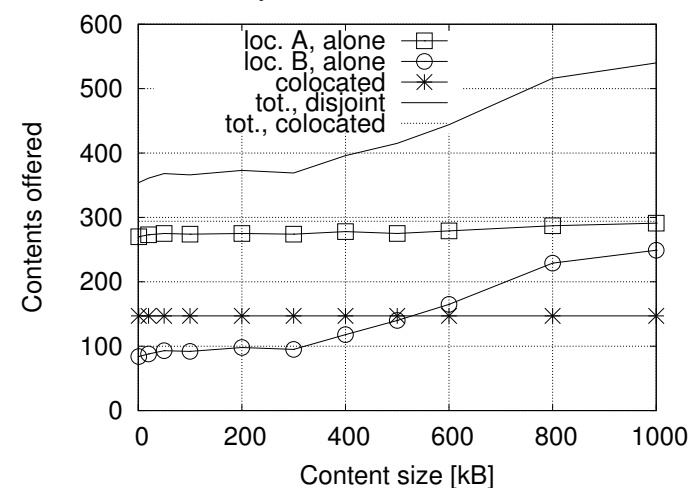

Fig. 5. Variable content size: number of offered contents as a function of the content size

the spillover benefits location $\mathrm{B}$, which again matches the performance of its competitor.

We next consider the effect of the imbalance between the flows in opposite directions in Fig. 4. We set $\lambda_{B}=0.05 \mathrm{veh} / \mathrm{s}$, and vary $\lambda_{A}$ between 0.1 and $2 \mathrm{veh} / \mathrm{s}$. The content size is again fixed at $500 \mathrm{kB}$ for each vehicle.

It can be seen that, as the rate of the left-to-right flow increases with respect to the opposite direction, location A becomes preferable. The newcomer's best choice is location $\mathrm{B}$ if $\lambda_{A}$ is below $0.5 \mathrm{veh} / \mathrm{s}$ (which matches the Colocation Overload (3.b) case). However, for higher rates, colocation becomes preferable for the newcomer, as it guarantees more successful transmissions, as predicted by the Colocation Overload (3.a) case. We also remark that, as expected, this NE is not socially-optimal, as can be seen by the much higher combined revenues in the disjoint case.

Finally, we look at the case of variable content size, which results in variable load offered to the RSUs. The arrival rates are fixed at $\lambda_{A}=0.5$ and $\lambda_{B}=0.05 \mathrm{veh} / \mathrm{s}$. Recall that if an OBU does not complete the transfer before leaving the RSU coverage, it will try afresh at the next RSU. This is shown in Fig. 5, where a sudden surge in offered traffic at location B can be detected for content sizes in excess of $300 \mathrm{kB}$.

A final look at Fig. 6 reveals that, for the chosen arrival rates, the content size increase does not affect the equilibria, but merely closes the gap between revenues achievable at disjoint locations.

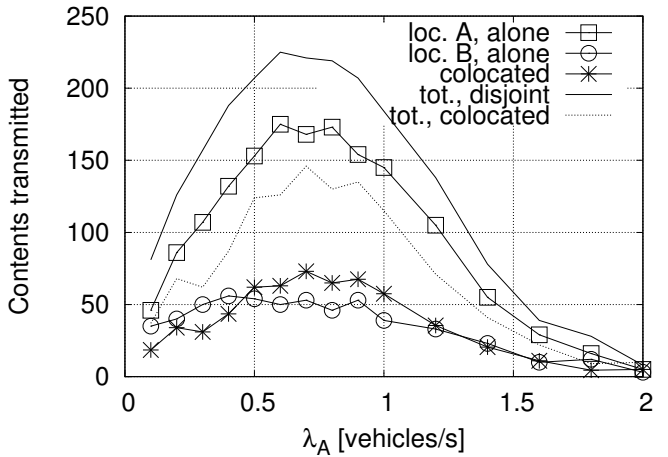

Fig. 4. Variable arrival imbalance: successful transmissions as a function of left-to-right vehicle flow intensity

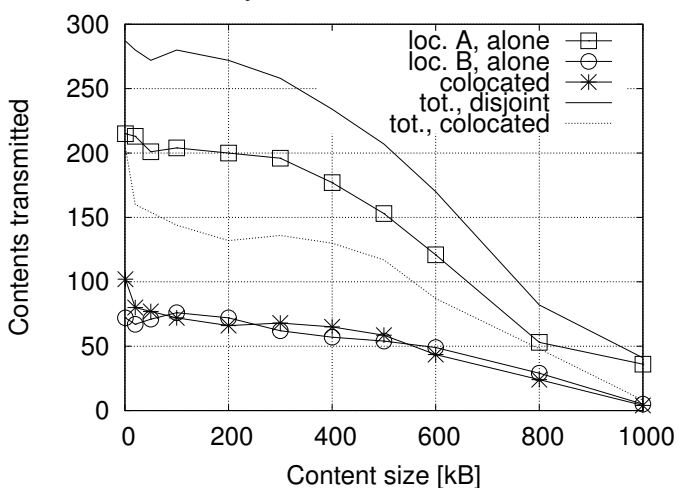

Fig. 6. Variable content size: number of successfully transmitted contents as a function of the content size

\section{ACKNOWLEDGMENT}

This work was supported by the European Union through the EU FP7 Euro-NF CAVE-NET project.

\section{CONCLUSIONS}

In this paper we looked at the problem of infrastructure deployment in VANETs through the lenses of game theory. We considered both simultaneous as well as leader-follower deployment, and quantified the inefficiency of equilibrium deployments compared to the social optimum. We then verified through simulations that, notwithstanding the necessary simplifications, our model correctly predicted the reachable equilibria as a function of traffic intensity.

\section{REFERENCES}

[1] G. Marfia, G. Pau, E. Giordano, E. De Sena, M. Gerla, "Evaluating vehicle network strategies for downtown Portland: opportunistic infrastructure and importance of realistic mobility models," MobiOpp, 2007.

[2] Y. Ding, C. Wang, L. Xiao, "A static-node assisted adaptive routing protocol in vehicular networks," ACM VANET, 2007.

[3] C. Lochert, B. Scheuermann, C. Wewetzer, A. Luebke, M. Mauve, "Data aggregation and roadside unit placement for a VANET traffic information system," ACM VANET, 2008.

[4] O. Trullols, M. Fiore, C. Casetti, C.-F. Chiasserini, J. M. BarceloOrdinas, "Planning roadside infrastructure for information dissemination in intelligent transportation systems," Computer Comm., vol. 33, 2010.

[5] Z. Zheng, P. Sinha, S. Kumar, "Alpha coverage: bounding the interconnection gap for vehicular Internet access," IEEE INFOCOM, 2009.

[6] Z. Zheng, Z. Lu, P. Sinha, S. Kumar, "Maximizing the contact opportunity for vehicular Internet access," IEEE INFOCOM, 2010.

[7] F. Malandrino, C. Casetti, C.F. Chiasserini, M. Fiore, "Content Downloading in Vehicular Networks: What Really Matters," IEEE Infocom Mini Conference, 2011. 